\title{
Population genetic history the red-necked stint
}

\author{
F. Kondrashov \\ Institute of Science and Technology Austria (IST Austria), Klosterneuburg, Austria \\ e-mail:fkondrashov@gmail.com
}

Key words: evolution, population genetics, polymorphisms, diversity, red-necked stint

Motivation and Aim: The nature of genetic changes contributing to species extinction and impeding population recovery remains poorly understood. How did living organisms become the way we know them today? This is the fundamental question that our laboratory is preoccupied with. We are less concerned with understanding the organisms themselves, our main focus is on how they evolved, that is how they changed over time. To address these fundamental issues we employ a diversity of modern tools. We apply mathematical modeling, we use available bioinformatic data and we perform our own experiments with a diversity of different model and non-model organisms.

Methods and Algorithms: We study the population genetic history of the critically endangered spoon-billed sandpiper and its sister species, the red-necked stint, which is of least concern.

Results: We found that while the red-necked stint population was relatively constant across 500,000 years, the spoon-billed sandpiper population peaked $15,000-25,000$ years ago during the last glacial maximum, when suitable breeding habitat was likely abundant, and has been declining since. The increase of the population prior to the ongoing decline led to accumulation of recessive deleterious polymorphisms, imposing a risk of inbreeding depression on the spoon-billed sandpiper population.

Conclusion: Thus, demographic changes leading to gain of deleterious genetic diversity pose an additional risk to species survival and recovery by increasing the cost of inbreeding. Specifically, species that had greater habitat availability during the last glacial maximum may be especially prone to this effect.

Acknowledgements: The participation at BGRS-2018 multiconference has been supported by Russian Ministry of Science project 28.12487.2018/12.1. 